the anesthesia may be fatal. Or, just as quiniu may cause so much breaking down of blood that the kidneys cannot functionate properly, so may the anesthetic seriously affect the heart or kidneys, or give rise to pneumonia.

To sum up, the question of quinin in malarial hemoglobinuria must be settled by careful clinical observation and experiment, and statements regarding it must be given credit in proportion to the accuracy with which the observations are made. In the meantime, quinin can be used cautiously, if parasites are present, giving the drug in a form most likely to be absorbed, in doses within the limit of ordinary safety, and stopping its administration as early as the microscope shows this to be proper. A temporary increase in the severity of the symptoms should not alarm the physician. It is not necessarily due to the drug. At the same time that the specific is being given, other methods of treatment must not be forgotten. The quinin is given to check the malaria, not for any particular symptom or complication. BIBLIOGRAPHX.

1. Golgi : Deutsche Med. Woch., 1892, Nos, 29-32.

2. Baccelli: Berliner Klin. Woch., 1890, p. 489

3. Williams. F. H.: Boston Med. and Surg. Journal, March 9, 1893 Heft 7 .

. Koch: Verhandiungen der deutschen Kolonial-Gesselschaft, 1897- 8 ,

5. Doering: Arbeiten aus dem Kais. Gesundheitsamte, Bd, xiv.

QUININ IN MALARIA.

IIIE INSATISFACTORY RESULTS OF ITS HYPODERMIC ADMINISTRATION.*

BY G. A. FACKLER, M.D.

CINCINNATI.

The application of medical knowledge is most gratifying when it results in accuracy of diagnosis and treatment. In the consideration of malaria we certainly have an opportunity to indulge in such gratification. The clinical picture, supplemented by manifestations on the part of the blood as presented by microscopic examination, furnishes the data for accurate diagnosis. The efficacy of quinin as a curative agent in the treatment of malaria has placed it among the small list of specific remedies. 'The comparative ease with which we can now diagnose a case of malaria and the confidence with which we can predict its defeat by quinin leaves only the consideration of the best and most reliable method of its administration.

Prior to the summer of 1898 I had occasionally resorted to the hypodermic administration of quinin in the treatment of malaria, but lack of brilliant results failed to stimulate me to the general adoption of this plan. The encouraging reports casually offered by some army surgeons who had resorted to the subcutaneous use of the drug in malarial patients of the Southern camps induced me to employ it in a series of cases which came under observation in the Cincinnati Hospital in the summer and fall of last year. Herewith, then, are offered the clinical facts elicited by a review of twenty cases, confirming the cursory previous observations made as to the unreliableness of quinin administered hypodermically in malaria. Refraining from a complete report of these cases such as would render them proper for publication, I submit an abridged statement of the salient features thereof, adapted to the time limit and your patience. The cases may readily be assigned to one of three classes: Three to the first class, successfully treated by hypodermic injection of quinin; 7 to the

* Presented to the Section on Practice of Mediciue, at the Fiftieth Annual Meeting of the American Medical Association, held at Columbus
Ohio, June 6-9. 1899. second class, successfully treated by quinin administered by the mouth; 10 to the third class, treated ineffectually by quinin hypodermically, but effectually by substitution of quinin by the mouth.

Classes 1 and 2 may be offered in sums total, Class 3 will be treated with sufficient detail to bring out the points that exemplify the object of this report in the most striking manner.

\section{CLASS I.}

Cases 1 and 2 were patients presenting all symptoms of double tertian fever, Case 3 a patient with a history of irregular chills and fever for three weeks, and blood examination dis. closing numerous crescents and ovoids-a total picture of an estivoautumnal type of malarial infection. In these three cases quinin bisulphate, gr. xv administered hypodermically twice daily, controlled the paroxysms within thirty-six hours, and, continued in gradually decreasing doses for a week, prevented a return.

CLASS II.

Cases 4 to 10, inclusive, were readily diagnosticated as double tertian. In all these cases the daily administration of gr. $\mathrm{xx}$ to $\mathrm{xxx}$ of quinin bisulphate, usually in morning and evening doses, controlled the paroxysms within twenty-four to fortyeight hours. In none did the daily temperature attain a height above 100 after twenty-four hours of quinin treatment and in none did the temperature rise above normal after seventy-two hours.

CLASS III.

Case 11.-James Scott, admitted Aug. 19, 1898, had paroxysms of chills and fever daily since August 15 .

Aug. 19. At 1:50 p.m., temp. 105. Intracorpsucular pigmented and non-pigmented plasmodia found in large numbers.

Aug. 20 . At 9 a.m., temp. 98.4 ; quinin, gr. $x$ hypodermically; at $12 \mathrm{~m}$., chill for twenty minutes; at 1 p.m., temp. 105.6 .

Aug. 21 . At 6 a.m., temp. 98.8 ; quinin bisulph., gr. $x$, at 9 a.m. hypodermically; at 12 m., temp. 105.8, preceded by chill at 11:20 a.m., quin. bisulph., gr. x, at 9 p.m. hypodermically.

Aug. 22. At 6 a.m., temp. 99; quinin bisulph., gr. $x$, at 9 a.m. hypodermically; 12 m., temp. 104.6, after chill; quinin bisulph., gr. $x$, hypodermically at, 9 p.m.

Aug. 23. At 6 a.m., temp. 99 ; quinin bisulph., by the mouth, 9 a.m.; 12 m., temp. 102.2 ; quinin bisulph. by mouth at 6 p.m.

Aug. 24. At 9 a.m. and 6 p.m., quinin bisulph. by mouth, gr. $x$; temp. not over 99 , normal throughout succeeding three days during which this treatment continued.

Case 12. - Harry Carr, admitted August 19, had been ill for six weeks, with irregular attacks of fever daily. His daily lowest temperature was about $12 \mathrm{~m}$. varying from 98.2 to 98.8 , rising with occasional chill.

Aug. 19. Temp. rose to 103.4 at 6 p.m.

Aug. 20. Temp. rose to 101.2 at 6 p.m.; quinin bisulph., gr. $\mathrm{x}$, hypodermically at $8 \mathrm{p} . \mathrm{m}$.

Aug. 21. Temp. 102 at 5 p.m.; quinin bisulph., gr. x, hypodermically at 5 p.m.

Aug. 22. Temp. 102.6 at 5 p.m.

Aug. 23. Temp. 102.6 at 5 p.m.

At 6 p.m., Aug. 23, and at 9 a.m. and 6 p.m., Aug. 24, quinin bisulph., was administered by mouth. As a result, the temperature never rose above 99. Treatment continued for three days.

Case 13.-George Wilson, admitted Aug. 20, had quotidian chill and fever during the preceding two weeks.

Aug. 20. Intracorpuscular pigmented and a few hyaline plasmodia. Lowest daily temperature, between 6 and 9 a.m., from 98 to 98.6 .

Aug. 21. Highest point reached was 102.2 , at 6 p.m.

Aug. 22. Quinin bisulph., gr. $x$, hypodermically at 10 a.m.; temp. rose to 104.6 at 6 p.m.

Aug. 23. Quinin bisulph., gr. xv, hypodermically at 12m.; temp. rose to 104.6 at $6 \mathrm{p} . \mathrm{m}$.

Aug. 24. Quinin bisulph., gr. $\mathrm{xv}$, hypodermically at $12 \mathrm{~m}$; temp. rose to 104.2 at $5 \mathrm{p.m}$.

Aug. 25. Quinin bisulph., gr. $\mathrm{xv}$, by mouth at $12 \mathrm{~m}$.; temp. rose to 101 at 3 p.m.

Thereafter quinin was administered at 6 a.m. and 6 p.m., in doses of gr. xv, and temperature normal.

Case 1\%.-Armstrong, admitted Aug. 29, had been ill for six weeks prior to admission. During the first three weeks he had chills and fever on alternate days, and thereafter quotidian. paroxysms.

Aug. 29. Found intracorpuscular pigmented hematozoa oc. cupying nearly the whole of the corpuscle. Lowest daily tem. perature at about 6 a.m., varying from 97.6 to 98.2 . Highest point reached was 101 , at 4 p.m. 
Aug. 30. Quinin bisulph., gr. $\mathrm{x}$, hypodermically at 10:30 a.m.; temp. 104.4 at $12 \mathrm{~m}$.

Aug. 31. Quinin bisulph., gr. x, hypodermically at 9 a.m.; temp. 105 at $12 \mathrm{~m}$.

Sept. 1. Quinin bisulph., gr. $\mathrm{x}$, hypodermically at 9 a.m.; temp. 98.4 at $12 \mathrm{~m}$.

Sept. 2. Quinin bisulph., gr, $\mathrm{x}$, hypodermically at 9 a.m.; temp. 105 at 3 p.m., and repeated the dose at 9 p.m.

Sept. 3. Quinin bisulph., gr. $x$, hypodermically at 9 a.m.; temp. 103 at 9 p.m. Repeated the dose at 9 p.m., and thereafter at 9 a.m. and 9 p.m.; quinin bisulph., gr. xv, by mouth, brought and held temperature at normal.

Case 15.-Chas. Stahl was admitted Sept. 16, with history of irregular attacks of chills and fever during preceding two weeks. Blood examinations were negative until Sept. 19, p.m., when large flagellate plasmodia were found. From Sept. 16 to Sept. 20 , temp. varied from 98 to highest between 6 and 9 p.m.- from 104 to 105.4 .

Sept. 20. At 9 a.m. and 9 p.m., quinin bisulph., gr. xv, hypodermically; temp. 9 p.m. 104 .

Sept. 21 . A. 9 a.m. and 9 p.m., quinin bisulph., gr. $x v$, hypodermicaly; temp. 9 p.m. 104.

Sept. 22. At 9 a.m. and 9 p.m., quinin bisulph., gr. $x v$, by mouth; temp. 99.8 at 6 p.m.

Sept. 23 . Triatment continued with temperature normal.

Case 16.-Albert Miers, admitted Sept 16, had onset of present illness the day before admission with chill and fever; lowest daily temperature between 3 and 9 a.m., varying from 98 to 99 . Highest temperature Sept 17, at 12 p.m., 103.8; Sept. 18, at 12 p.m., 103.6 : Sept. 19, at 12 p.m., 102.2. Blood examination showed intracorpuscular bodies.

Sept. 20. Quinin bisulph., gr. $x$, hypodermically, at 9 a.m. and 6 p.m.; temp. 102 at 9 p.m.

Sept. 21. Quinin bisulph., gr. x, hypodermically, at 9 a.m. and 6 p.m.; temp. 102.2, 9 p.m.

Thereafter quinin by mouth, gr. $\mathrm{x}$, twice daily, with total subsidence of symptoms; no elevation of temperature over 99.2. Case 1\%.-H. Herberg, admitted Sept. 19, with history of chills and fever daily for several weeks. Sept. 20, blood examination showed intracorpuscular pigmented bodies; lowest daily temperature between 3 and 6 a.m. varving from 97.4 to 98 .

Sept. 20. Highest point attained 105, at $12 \mathrm{~m}$.

Sept. 21. Quinin bisulph., gr. xv, at 9 a.m.; temp. to 104.4 at 1 p.m.

Sept. 22. Quinin bisulph., gr. xv, at 9 a.m. and 6 p.m.; temp. to 105.4 at $12 \mathrm{~m}$.

Sept. 23. Quinin bisulph., gr. $x v$, at 9 a.m.; temp. to 104 at $12 \mathrm{~m}$.

Sept. 23. At 6 p.m. and Sept. 24 at 9 a.m., quinin bisulph., gr. xv, by mouth; temp. to 100.2 at 9 p.m.

The same treatment continued resulted in elevation of temperature Sept. $2 \tilde{5}$, to 100 at 6 p.m.; thereafter normal.

Case 18. - Jacob Jauch, admitted Sept. 19, with history of chills and fever for two days preceding admission. Sept. 19, small hyaline intracorpuscular bodies found; lowest daily temperature at about, 6 a.m., varying from 100 to 102.

Sept. 19. Highest temperature at 3 p.m., 106.

Sept. 20. Highest temperature at 9 p.m., 106. Quinin bisulph., gr., xv, hypodermically at 9 p.m.

Sept. 21, 22. 23. Quinin bisulph., gr. xv, hypodermically, twice daily; temp. rising with chill each day between 3 and 6 p.m., to 104.8 to 105.5 .

Sept. 24. Resorted to administration of same doses of quinin by mouth, morning and evening, and continued this method with following result.

Highest temp. on Sept. 24, 103; Sept. 25, 101; Sept. 26, 99; thereafter normal.

Case 19.-Schwenk, admitted Sept. 21, had chills and fever daily for about two weeks. On day of admission numerous pigmented and nonpigmented intracorpuscular bodies were found; lowest daily temperature at 3 to 6 a.m., from 97.8 to 98.2.

Sept. 21. Temp. at 3 p.m., 105; quinin., gr. xv, hypodermically, at 6 p.m.

Sept. 22. Quinin, gr. xv, hypodermically at 9 a.m and 6 p.m.; temp. at 3 p.m., 104.6.

Sept. 23. Quinin, gr. $x r$, hypodermically at 9 a.m. and 6 p.m.; temp. at. 3 p.m., 104.

Sept. 24. Quinin, gr. $\mathrm{xr}$, hypodermically at 9 a.m.; temp. at 3 p.m. 102.6; quinin by mouth at 6 p.m., and thereafter at 9 a.m. and 6 p.m., with abeyance of all symptoms on Sept. 25 .

Case 20.-Selcer, admitted Sept. 21, onset of illness indefinite.

Sept. 22. Numerous crescents and ovoids and some hyaline intracorpuscular bodies were found; estivoautumnal; lowest daily temperature at about 6 a.m., about 99.

Sept. 21 . Temp. to 03 at 12 p.m.
Despite hypodermic administration of quinin bisulph., gr. $\mathrm{xv}$, at 9 a.m. and 6 p.m. on Sept. $22,23,24$, temp. rose to 103 to 104.2 between 9 and 12 p.m.

Sept. 25. Order was changed to quinin by mouth, in same dose and at same interval. Temp. highest Sept. 25 at 9 p.m., 99.4 , and thereafter normal.

A general résumé of the 20 cases furnishes the following data: In 3 cases quinin hypodermically was successful; in 10 cases quinin was not successful; in 7 cases quinin by the mouth was successful; in 10 cases quinin by the mouth was successful after hypodermic administration had failed-a total of 17 cases in which quinin by mouth. was successful as a curative measure in the treatment of malaria. In only 3 out of 13 cases, in which the remedy was introduced subcutaneously was an abeyance of symptoms secured. This experience was certainly at variance with that reported casually by some of our army surgeons.

It is but natural that we should seek for a rational explanation of this fiasco of hypodermic medication. We were assured of the correctness of the diagnoses, for no cases were designated as malarial unless the blood examinations furnished evidence which would warrant the diagnosis. All compounds of quinin, or better said, all salts of the alkaloid, have a toxic effect upon the malarial organism, and hence a curative action when administered in malarial diseases. The failure cannot be attributed to the compound employed, per se. The beneficial effect of the same preparation from the same stock when given by the mouth dispelled all doubt as to the therapeutic power of that compound. The procedure adopted for injection and the site selected were in perfect accord with the plan followed by most clinicians and observers. Where then was the defect in the inherent curative value of the method? The therapeutic failure of any remedy can not be ascribed to it essentially unless positive evidence of its absorption is furnished. It was a noticeable observation in all of our cases in which hypodermic medication was resorted to that after the majority of injections, the patients complained of pain in the region implicated by the injection. In some instances a distinct deep-seated hardness of tissue persisted for days after the treatment. It is a conjecture, in all probability bordering upon a certainty, that the solution, while dilute, as it must be with the use of the bisulphate, causes decided irritation of the tissues with which it comes in contact. The resultant localized disturbance of the circulatory conditions of the parts involved interferes with the absorptive power of the area and thus the remedy creates a bar to its own ab. sorption.

If the observations thus submitted to you are indicative of the general experience among a larger number of cases with the same or other compounds of quinin we are forced to the assumption that the hypodermic use of quinin in malaria is never indicated except in cases in which stomach and bowels reject the dose ingested or injected. Unless my observations and deductions are erroneous, because of the uncertain action of the hypodermic injection of quinin, its administration by the mouth should be adopted in all cases.

\section{DISCUSSION ON PAPERS OF DRS. DOCK AND FACKLER.}

Di. Charles G.Stockton of Buffalo.--It seems to me the papers are most timely. For some time there has been growing confusion as to the exact place of quinin in the treatment of malaria. Now, in the matter of the time for the administration of quinin by the mouth, as suggested by Dr. Dock, I beg to take exception. There should be no rule of time for all individuals alike; it requires a study of each individual case to determine at what particular time the drug will affect the plasmodium. It depends too upon the primary digestion of individuals. In some instances the effect of the drug is ex- 
ercised in the course of an hour. Dolge's method of giving quinin prcvious to the paroxysm has not met with the expected success.

Cinchonism is found to be more intense if the quinin shows an ellect, and yet the paroxysm still comes on. Quinin is said to be difficult of administration; all will not agree with the writer; it is easy of administration. I question the hypo. dermic use of quinin mentioned by Dr. Fackler; I have had experience during the past year and I have come to the same conclusion that he has reached. In one case there was failure to relieve the paroxysm by hypodermic use of the drug, but the patient was relieved by the administration of the drug by the mouth. In that case, as well as in two others, there hap. pened troublesome abscesses at the seats of injection, which was something $I$ had never seen before. The local irritation probably accounted for the slow absorption of the quinin and the non-relief of the symptoms.

Dr. JUdson Daland of Philadelphia.-I wish to express my pleasure in hearing the paper and wish to speak in regard to two points. So far as the use of quinin is concerned, my experience has taught me that two things are to be considered -does malaria exist, and is quinin absorbed? Exclusive of hospitals, experience teaches that less than half the cases diag. noserl as malarial are truly malarial. I am speaking of the eases that come in the ordinary course of practice, and not the cases that come under close observation in hospitals. I was especially pleased with the statements made in reference to the quantity of quinin to be employed, and also as to the time it should be administered. Personally, I am distinctly of the opinion that the bichlorid of quinin is the preferable salt, on account of its solubility and the fact that the solution is reliable. So far as the dose is concerned, I agree with the statements made by Dr. Dock. As to the time of giving the dose it seems to me that one particular point should be considered, i. e., the time in the life eycle of the plasmodium when it is most susceptible to the action of quinin. In other words, I am inclined to the opinion that the time for giving the quinin is soon after the violence of the paroxysm has passed over. This corresponds to the time that there is a maximum quantity of free ameboid bodies in the blood. It is highly probable that when the malarial parasite enters the red blood-cells, quinin circulated in solution in the plasma could not exert its effects so completely as when the parasite is free in the blood. This particular time accords well with the observations of Dr. Stockton and $I \mathrm{am}$ sure that benefit is to be obtained from a preliminary course of calomel. After the paroxysm there is an increase in the absorption of the cinchona salt. It is interesting to know that non-absorption of quinin is a common occurrence.

While observing a case of ordinary tertian intermittent fever the hematokrit showed a loss of 10 per cent. of red bloodcells as the result of a single paroxysm of chill, fever and remission.

Ifemoglobinuria is a rare occurrence. I recollect one specimen sent me from Louisiana. Hcmoglobin alone was present, but upon examining the sediment, a few shells of red blood corpuscles could be seen. I also recollect another case in which the albuminuria increased during each sueceeding paroxysm of the attack.

Dr. F. S. JoHnson of Chicago.-The absorption of quinin is very uncertain. It is impossible to treat in any other way than by hypodermic injection or by inunetion and be sure of your results. I will relate one case as an illustration of this. A man who had been very strong and who became extremely weak, was given quinin by the mouth without effect. Hypodermic injections of large doses of quinin were given, 40 to 60 grains by deep in jection, and soon the malaria subsided and the patient got well. He was so weak at the time that it was impossible for him to rise from the bed. The hypodermic injection was made by deep puneture. Quinin given subeutaneously may cause sloughs; but if deep injections be given there will be no sloughs or abscesses if the solution used be sterile. In many cases quinin by the mouth has a different effect from that given in some other way. I saw a lady with well-pronounced intermittent fever, not of severe type. When she took quinin by the mouth she had delusions and hallucinations. She took it several days without effect, but when the fever began to respond to the quinin and the paroxysms became less severe she had dreams. She recognized them as dreams, but one morning she thought that all the dreams she had had were real. The quinin was stopped and the hallucinations subsided, but then the fever returned. I was in a quandry as to what treatment to give. The rectum was irritable, so I could not treat her in this way. She was a highly nervous, sensitive woman and I did not dare to propose the use of this method. I then gave her from 150 to 200 grains of quinin mixed with equal parts of oleic acid and one-third the amount of coca butter, and this was rubbed in daily. The temperature then came down and did not return, nor did she have any more hallucinations. Quinin by the mouth has a different effect from that when given by the skin, by inunctions or hypodermatically. In another case of a returned soldier the quinin was given by inunetion; 200 grains were given daily. He could not take the drug by the mouth or rectum, for it would not be absorbed. He was afraid to take the injections through fear of abscesses. He took it by the skin in this way, and his recovery was very prompt.

Dr. H. A. HARE of Philadelphia.--It seems to me the discussion of an important subject illustrates one of the most im. portant points. The readers of the papers and the discussers, including myself, are men who may have less opportunity of studying the conditions now discussed than many of the gentlemen in the audience. I think it is rather unfortunate that physicians who have large opportunities credited to them have not done more toward the study of this subject.

What has been said regarding the absorption of the drug is true. I think one of the greatest questions connected with the therapeutics of quinin is regarding the time to give the drug; physicians are not sure of the alimentary canal, whether it is in condition to receive the drug. Quinin is precipitated by the juice of the stomach, and it is often found that it will not be absorbed at all. The use of mercurials and acids as resorted to in the South is exceedingly advantageous. I am inclined to believe that many physicians, not having been trained in the use of the microscope, often make a diagnosis of this disease when malaria is not present at all; I often come across cases illustrating this point. Again the diagnosis is often made of malarial fever, mild in character, which is not malaria at all. I would like to reiterate the fact that where the clinical course is like certain types of malarial fever a blood examination alone will prove the diagnosis to be correct.

It is unfair to Dr. Dock to refer to a certain method as the Gody's method. My experience has taught me that this is the proper time to give the quinin, and I have used it months with great success.

One other point regarding the hypodermic administration of the drug; this is most useless. I make a difference between the hypodermic method and the intravenous injections. There is great difficulty in absorption except in an acid medium. Quinin in the tissues is precipitated by the alkaline juices of the tissues. The quinin is precipitated and is immediately surrounded by an infammatory exudate and is absorbed like catgut ligature. The drug should be injected into the veins; the medium in which it is carried is a normal saline solution, and it should be injected slowly. There is one important point that physicians should remember; that is that when quinin comes in direct contact with the heart it is terribly depressing; in animals it acts in the same way as in man. In a Newfoundland $\operatorname{dog}$ quinin was injected into the reins and the heart stopped at once. IJo not forget the point that large doses are depressing to the heart, and may do the patient much damage.

Dr. DUNHAM of Cincinnati.-I wish to make a plea for the use of the hypodermic syringe. There is a salt of quinin which is sometimes overlooked, and that is a double salt put up by Irerek. It is soluble in equal parts of water, and can be in serted in small quantities between the gluteal fold. This injection will be absorbed, and cases will yield readily if it can be kept out of the muscles, and the inflammation kept down. All cases of malaria need not be treated by hypodermic injection, but only those cases where it is impossible to get quinin into the system in any other way. The great trouble seems to be in finding out when to give the quinin, and whether you have not something else to contend with. There is nothing nicer to give with quinin than calomel or a little acid; I prefer the ealomel. The time of giving quinin is very important-that is, the time of getting quinin into the blood. If quinin is brought into the blood at one time it is one thing; but if at another time, it is another. If the malarial organism is surrounded by red blood-corpuscles it is difficult to get the quinin to act upon the organism. If the quinin is introduced after segmentation, good effects will follow. In the tertian or quartan forms you do not get the full effects in the first day; it is best to eatch the organisms as they are segmenting. By using the double salt of quinin you can place in the blood your drug at the time it is needed. The pain in using the hypodermic injection is very greatly reduced-almost to a minimum-by using the double salt of quinin, using two parts of water, and by keeping between the deep gluteal muscles.

Dr. J. C. Wirson of Philadelphia spoke of his experience im the German Hospital of Philadelphia with reference to outbreaks of malarial disease latent for weeks and months, in soldiers who had served in Cuba and Porto Rico. Many of these cases had stubbornly resisted quinin by the mouth, even 
in large doses and administered under most varied circumstances, but yielded to moderate doses by the hypodermic method-in some instances the double salt of quinin and urea being used; sometimes the hydrochlorate of quinin.

With reference to the precipitation of the quinin salts in the alkaline fluid of the subcutaneous tissues our knowledge is somewhat limited. If precipitated it is certainly redissolved and absorbed, as shown by unquestioned therapeutic results. If precipitated under these circumstances, it is equally liable to be precipitated in the blood when introduced into the veins.

The cases of Panama and Chagres fever, at one time common in our coast-wise vessels, usually admitted to the hospital comatose, yielded promptly to large doses of quinin hypodermically and by the rectum.

Dr. GEORGE Dock of Ann Arbor, Mich.-In order to make my paper short I left out all else except that which referred to quinin alone. Individuals differ, and a great deal is often required besides the giving of quinin. Regarding Dr. Fackler's paper, he has given strong evidence of what a number of observers have stated. In all cases of Dr. Fackler's, quinin was given at a time when it was less likely to do good. In regard to the hypodermic injection I only wish to state that the hypodermic method, in my opinion, is necessary in many cases. Deep injections are better than strong injections. If given in the museles there is less pain and less tendency to the formation of abscesses. In any case the salt of quinin is apt to give pain and to cause abscess; these facts cannot be lightly turned aside.

1) R. ( $x$. A. FACKLER, in conclusion, wished to be positive that his statement in regard to the method of quinin injection was not misconstrued. In every case the deep injection was resorted to. In no case was an effort made to administer the remedy at a time coincident with a certain phase of the fever, and, ret, to some of our patients it was accidentally given in aceordance with the sugrestions of Dr. Dock. The question of the proper time for the exhibition of quinin in malaria is still sub judice and will probably never be settled to the satisfaction of all. The preceding discussion illustrates the various opinions entertained as to the absorption of quinin. It is universally acknowledged that the conditions of the digestive tract and of the circulatory organs play an important role in the absorption of the drug. The amount absorbed and the period required for this act must vary with the condition of the part into which it is introduced. Hence the utter folly of predicting the positive action of quinin within or at a specified period of time after its administration by mouth or injection. Much more rational is the introduction by either method into the body, of a dose, at stated intervals in the course of the day so as to insure the presence of a therapeutically effective amount of quinin in the circulating media at all times during that day. This plan was followed in all our cases and the results secured were certainly practical illustrations of its efficacy. I desire finally to call attention to the dearth of detailed reports of series of cases treated by the hypodermic injection of quinin and the uncertainty of the value of observations made upon one case by one individual. It would certainly be desirable that those who have made general assertions as to the efficacy of this method in the treatment of malaria should contribute specific reports of their cases to the literature upon the subject.

\section{EXSTROPHY OF THE BLADDER** BY C. A. WHEATON, M.D.

$$
\text { ST. PAUL, MINN. }
$$

None of the congenital defects of man have elicited a greater diversity of opinion as to etiology, or more difficulty in correction than those affecting the genital extremity. Until a comparatively recent date the deformities of the genitalia of the male and female offspring were attributed to failure in the evolution of the structures which go to make up the distinctive characteristics of sex.

Embryology teaches us that the allantois is the fetal structure from which is derived the bladder and its suspensory ligament; that the urethra is primarily a groove opened beIow, which gradually advances from the urogenital sinus, and from the imperfect closure of this groove and the imperfect elaboration of this allantoic structure arise the defects which I have selected to here

* Presented to the Section on Surgery and Anatomy, at the Fiftieth Annual Meeting of the American Medical Association, held at Columbus,
Ohio, June 6-9,1899. discuss briefly. The latest, and perhaps the best, résumé of this subject is to be found in an article written by $\mathrm{F}$. Tilden Brown ${ }^{1}$. In this he leads us to understand that the etiologic factor in the production of hypospadis and epispadias is practically the same, that "both conditions are due to the rupture of the urethra in consequence of urinary retention by the absence or retarded formation of the glandular urethra."

"The usual theory of epispadias is similar to that which explains hypospadias as due to incomplete or faulty development of the parts affected. Thus, Thiersch regarded the malformation as due to a pelvic closure and division of the cloaca, which was faulty as regards time. Under normal circumstances pelvic closure occurs first, so that the corpora cavernosa, developing in connection with the rami of the pubes, are already agglutinated to the sexual buds before the sinus urogenitalis is pushed forward by the developing perineum. But if the cloacal division occurs before pelvic closure, the two halves of the corpora cavernosa are not united; the urogenital sinus is pushed forward between them, and they unite below instead of above it. This hypothesis evidently depends for its premise on the ausence of the symphysis, and, unfortunately for it, the symphysis is not always absent. On the other hand, a number of facts undoubtedly favor the rupture hypothesis, and are quite inexplicable on any theory of defective development. Absence of imperviousness of the urethra leads to accumulation of urine in the bladder and dilatation of that organ; a rupture occurs at a time when the abdominal envelopes are not yet quite perfect, and this rupture may affect the entire urinary sac from the navel down, or only its lower part. So we get epispadias with exstrophy of the bladder, or simple epispadias. Thus, Thiersch has proved, both in the dead and living subject, that the ureters are much dilated in all these cases; he even succeeding in passing a No. 6 English catheter through the ureters into the pelvis of the kidney. Scar tissue also is found all around the bladder opening, as from an unsuccessful plastic operation-distinct evidences of rupture and cicatrization.

"But the most incontestable proof of the correctness of this so-called mechanical theory of the origin of epispadias is found in the cases of intra-uterine healing of exstrophy of the bladder and of epispadias, of which the best case is that of Kuster. Here, in a boy 1 year and $\%$ months old, an unmistakable scar stretched from the umbilicus on to the dorsum of the penis. In fact, it may well be that the separation of the pubic rami is the direct effect of the same urinary retention that causes rupture of the vesical and urethral walls. Thus, an early rupture of the urethra would give us a simple epispadias with normal symphysis, whereas, if this early rupture does not occur, the symphysis separates; if the urethra now ruptures, we get simple epispadias with absence of symphysis. If both bladder and urethra rupture we get exstrophy of the bladder and epispadias."

It has been unquestionably demonstrated that the excretion of urine is active during the latter months of fetal life, and that the bladder undoubtedly fills and empties itself frequently during that period, and a careful review of the literature of the subject leads me to believe that this is the correct explanation of these peculiar caprices of Nature. It is not my purpose to discuss the causes of these defects; but to submit for your consideration the following cases which seem to me to be of special interest.

1 Disease and Injuries of Urethra; Morrow's "Genito-Urinary Dis 